\title{
Prácticas evaluativas y significados evaluados por profesores del bachillerato mexicano sobre la noción de ecuación lineal
}

\author{
Evaluative practices and meanings evaluated by the \\ Mexican high school teachers on the notion of linear equation
}

\author{
Raúl Alonso Ramírez Escobar ${ }^{1}$ \\ Silvia Elena Ibarra Olmos \\ Luis Roberto Pino-Fan ${ }^{3}$
}

Resumen: La evaluación del aprendizaje es un proceso que teóricamente debe contribuir en la formación de los estudiantes y en la mejora de las prácticas docentes, posicionándose con el paso del tiempo como uno de los procesos más complejos en el ámbito educativo. Esta es quizá una de las razones por las que poco se ha avanzado en su desarrollo, particularmente en el caso de la Educación Matemática. Con la intención de conocer más sobre dicho proceso, el propósito de esta investigación fue caracterizar las prácticas didáctico-matemáticas que, sobre evaluación del aprendizaje, manifiestan profesores de bachillerato en México cuando enseñan la noción de ecuación lineal. Al mismo tiempo, se indagó respecto a los significados efectivamente evaluados sobre dicha noción matemática. Los resultados evidencian que factores tales como: el currículo nacional, normativas institucionales, así como la formación y el conocimiento de los profesores sobre el tema matemático en cuestión y la correspondiente evaluación

Fecha de recepción: 26 de diciembre de 2017. Fecha de aceptación: 28 de enero de 2019

${ }^{1}$ Departamento de Ciencias Básicas. Tecnológico Nacional de México, Instituto Tecnológico de Nogales, raul.re@nogales.tecnm.mx, orcid.org/0000-0002-6280-0957.

2 Departamento de Matemáticas. Universidad de Sonora, México, sibarra@matuson.mx, orcid.org/00000002-1344-2516.

${ }^{3}$ Departamento de Ciencias Exactas. Universidad de Los Lagos, Chile, luis.pino@ulagos.cl, orcid.org/00000003-4060-7408. 
de su aprendizaje, inciden directamente en las prácticas evaluativas de los profesores y en los significados que evalúan.

Palabras clave: evaluación del aprendizaje, ecuación lineal, significados del objeto matemático, bachillerato.

\begin{abstract}
The evaluation of learning is a process that theoretically should contribute to the formation of students and the improvement of teaching practices, positioning itself over time as one of the most complex processes in the field of education. This is perhaps one of the reasons why little progress has been made in its development, particularly in the case of Mathematics Education. With the intention of knowing more about this process, the purpose of this research was to characterize the didactic-mathematical practices that, on assessment of learning, manifest high school teachers in Mexico when they teach the notion of linear equation. At the same time, we inquired about the meanings evaluated on this mathematical notion. The results show that factors such as the national curriculum, institutional regulations, as well as the training and knowledge of teachers on the subject in question and the corresponding evaluation of their learning, directly affect the evaluative practices of teachers and meanings that evaluate.
\end{abstract}

Keywords: evaluation of learning, linear equation, meanings of mathematical object, baccalaureate.

\title{
1. ANTECEDENTES
}

La literatura sobre evaluación educativa pone de manifiesto que actualmente la evaluación del aprendizaje está considerada una parte importante del proceso de instrucción, pues proporciona información para mejorar la práctica docente, incidiendo directamente sobre los procesos de enseñanza y aprendizaje, las tareas, los materiales, la organización, la planificación, etc., así como sobre el progreso de los alumnos hacia los objetivos de aprendizaje (Moya, 2001; Wormeli, 2006; De Vincenzi y De Angelis, 2008; Harlen, 2012; Moreno, 2012).

No obstante, diversos autores (e.g., Webb, 1992; NCTM, 2000; Calderón y Deiros, 2003; Becerra y Moya, 2008; Hernández, 2013; Álvarez y Blanco, 2014; 
Dolores y García, 2016) señalan que en la evaluación del aprendizaje en matemáticas sigue prevaleciendo una práctica evaluativa centrada, fundamentalmente, en la aplicación de exámenes escritos de formatos cerrados que sancionan o certifican lo que, supuestamente, el estudiante debió aprender. En dichos exámenes se valora el conocimiento matemático aprendido restringiéndolo a la reproducción de definiciones, conceptos y algoritmos; en estos términos la evaluación realizada no garantiza al estudiante un avance productivo, toda vez que debería involucrar aprendizajes, enseñanza, acción docente, currículo y aspectos institucionales, entre otros elementos. De acuerdo con Goñi (2008), difícilmente se avanzará hacia una enseñanza más eficaz de la matemática si no se modifican las prácticas de evaluación.

En el caso del bachillerato, nivel educativo que cuenta desde 2008 con un Marco Curricular Común a través del cual se promueve un modelo de Educación Basada en Competencias (EBC), en México se han implementado una serie de acciones orientadas a establecer una nueva visión sobre la evaluación del aprendizaje. En documentos oficiales, lineamientos y acuerdos secretariales, se establecen consideraciones sobre la manera en que los profesores deben llevar a cabo el proceso de evaluación del aprendizaje en sus aulas, así como sugerencias para el posible uso e implementación de diferentes instrumentos de evaluación. Sin embargo, la información presentada en tales documentos es muy genérica, pues no se hacen explícitos ni se distinguen planteamientos relacionados con el área de matemáticas.

Los profesores de matemáticas, como representantes de su institución, deben establecer una estrategia para llevar a cabo la evaluación del aprendizaje de sus estudiantes, apoyados en el programa oficial de la asignatura (currículo). Entonces, dicho sistema de prácticas evaluativas podría estar siendo influenciado por normas institucionales e incluso por sus propias creencias y concepciones sobre la evaluación (Dolores y García, 2017).

En este contexto, este documento tiene por objetivo presentar los resultados de un estudio llevado a cabo para explorar el tipo de prácticas evaluativas e instrumentos que utilizan los profesores de bachillerato en México, al evaluar la noción de ecuación lineal. Además, se indagan los significados de dicha noción que son efectivamente evaluados con tales prácticas.

En el siguiente apartado se presentan las nociones teóricas que sustentan el estudio y que permitieron efectuar los análisis correspondientes. Posteriormente se señala la metodología seguida en cada una de las etapas de esta investigación. En el tercer apartado se presentan los resultados obtenidos a partir del 
estudio sobre los diferentes significados, es decir, los significados pretendidos por el currículo de los dos diferentes subsistemas de bachillerato en los que laboran los profesores bajo estudio, así como los significados pretendidos, implementados y evaluados por dichos profesores. Finalmente se presenta una reflexión sobre la representatividad del significado holístico de la ecuación lineal en los significados señalados en el apartado anterior.

\section{MARCO TEÓRICO}

El marco teórico empleado en esta investigación fue el Enfoque Onto-Semiótico (EOS) del Conocimiento y la Instrucción Matemáticos (Godino y Batanero, 1994; Godino, Batanero y Font 2007), el cual consiste en una teoría inclusiva que proporciona herramientas para el análisis didáctico de procesos de instrucción matemática. A continuación se describen las herramientas que fueron utilizadas.

Dentro del EOS, la noción de sistema de prácticas es fundamental desde un punto de vista epistemológico y didáctico, pues se refiere a "toda actuación o manifestación (lingüística o no) realizada por alguien para resolver problemas matemáticos, comunicar a otros la solución, validar la solución y generalizarla a otros contextos y problemas" (Godino, Batanero y Font, 2007, p. 127). Estas prácticas pueden ser desarrolladas por una persona o compartidas por una institución. El sistema de prácticas operativas y discursivas que realiza una persona para resolver un determinado campo de problemas (del cual emerge el objeto matemático en cuestión), constituye el significado personal de dicho objeto matemático. Si el sistema de prácticas es realizado por una institución (conjunto de personas inmersas en una misma clase de situaciones-problemas), éste constituirá el significado institucional de dicho objeto (Godino y Batanero, 1994).

Con relación a los significados institucionales, el EOS propone los siguientes tipos: a) implementado, sistema de prácticas efectivamente implementadas por el docente; b) evaluado, subsistema de prácticas que utiliza el docente para evaluar los aprendizajes; c) pretendido, sistema de prácticas incluidas en la planificación del proceso enseñanza-aprendizaje; y d) referencial, sistema de prácticas que se usa como referencia para elaborar el significado pretendido (por ejemplo, las descritas en el currículo). De acuerdo con Pino-Fan, Castro, Godino y Font (2013, p. 147), "en una institución de enseñanza concreta este significado de referencia será una parte del significado holístico del objeto matemático". Asimismo, el EOS 
también propone considerar los siguientes tipos de significados personales: a) global, la totalidad del sistema de prácticas personales que es capaz de manifestar el sujeto relativas a un objeto matemático; b) declarado, prácticas efectivamente expresadas a propósito de las pruebas de evaluación propuestas, incluyendo tanto las correctas como las incorrectas desde el punto de vista institucional; c) logrado, prácticas manifestadas conforme a las pautas institucionales.

Este enfoque teórico considera a los objetos matemáticos como entidades intervinientes y emergentes de los sistemas de prácticas realizadas al resolver un campo de problemas (Godino y Batanero, 1994). En ese sentido, cuando un individuo ejecuta una práctica matemática, activa un conglomerado formado por seis tipos de objetos matemáticos primarios (situaciones-problemas, lenguajes, conceptos, proposiciones, procedimientos y argumentos), los cuales conforman una configuración ontosemiótica (Godino, Batanero y Font, 2007; Pino-Fan, Godino y Font, 2015). Esta configuración ontosemiótica puede verse desde una perspectiva epistémica (si la configuración está asociada a las prácticas matemáticas desarrolladas por una institución o representante de la misma) o cognitiva (si la configuración está asociada a las prácticas matemáticas desarrollada por un estudiante a propósito de la solución de un problema).

Tal como señala Pino-Fan (2014, p. 347), "el modelo ontológico y epistemológico propuesto por el EOS revela la complejidad inherente a los significados (conocimientos) institucionales y personales que se ponen en juego en la enseñanza y aprendizaje de las matemáticas". En este sentido, en cierta forma, el objetivo de los profesores en un proceso de enseñanza-aprendizaje debería ser el cautelar: que las configuraciones cognitivas (que movilizan los estudiantes en sus prácticas matemáticas) se adapten progresivamente a las configuraciones epistémicas (que moviliza el profesor en su práctica de enseñanza). Consecuentemente, se puede decir que en el EOS se entiende la evaluación desde un punto de vista formativo y, que refiere al proceso de observación sistemática del progreso cognitivo de los estudiantes. En este sentido, aunque actualmente en el EOS no se tiene una definición explícita sobre lo que significa la evaluación, para efectos de este estudio entenderemos que la evaluación refiere al proceso de negociación de significados (de un objeto matemático) que realiza el profesor con sus estudiantes y, cuyo objetivo fundamental es lograr un acoplamiento progresivo entre las configuraciones ontosemióticas cognitivas que ponen en juego los estudiantes en sus prácticas matemáticas y las configuraciones ontosemióticas epistémicas que pone en juego el profesor en su práctica de planificación e implementación (significados pretendidos e implementados). 
En este estudio se asumió la posible presencia de prácticas evaluativas en dos direcciones: durante el proceso (evaluación formativa), y al cierre del proceso (sumativa). La evaluación sumativa se entenderá en términos de lo señalado por Casanova (2007), como el tipo de evaluación que orienta a verificar el cumplimiento de los objetivos y estándares previamente determinados en el programa, lo que permite, además, cumplir con la función de control y acreditación del aprendizaje. Casanova (2007) describe que la evaluación formativa cumple una función reguladora de los procesos de enseñanza y de aprendizaje, lo cual permite llevar a cabo ajustes y adaptaciones progresivas durante el curso porque se centra, más que en los resultados del aprendizaje, en los procesos que se ponen en juego para el logro de tales resultados. Así también, la evaluación formativa requiere que el profesor y el estudiante manifiesten una comprensión compartida de los objetivos del aprendizaje (Shepard, 2000; Stiggins, 2001).

Para el análisis de los procesos instruccionales se utilizaron las nociones de configuración ontosemiótica epistémica y trayectoria epistémica (Godino, Contreras y Font, 2006). En cada caso identificando la red de objetos que intervienen y emergen de los sistemas de prácticas institucionales, esto es, cómo van apareciendo y relacionándose estos objetos matemáticos primarios. El conjunto de configuraciones epistémicas, secuenciadas en el tiempo didáctico, conforma la trayectoria epistémica. Otra herramienta teórico-metodológica del EOS que fue clave para sustentar la investigación, fue la noción de trayectoria docente, entendida como la secuencia de actividades que va efectuando el profesor durante un proceso de enseñanza-aprendizaje. El segmento de la trayectoria docente, referida al accionar del docente tanto antes como durante y después de conducir un proceso de enseñanza-aprendizaje, alrededor de una situación-problema, se denomina configuración docente (Godino, Contreras y Font, 2006). En dichas trayectorias es posible clasificar cada una de las acciones docentes según su función, es decir: planificación, motivación, asignación, regulación, evaluación e investigación.

\section{METODOLOGÍA}

El estudio se realizó bajo el paradigma de investigación cualitativa (Sandín, 2003). Esta metodología permitió realizar "descripciones detalladas de situaciones, eventos, personas, interacciones y comportamientos que son observables, incorporando la voz de los participantes, sus experiencias, actitudes, creencias, pensamientos y reflexiones tal y como son expresados por ellos mismos" (Ibíd., p. 20). 
La investigación se llevó a cabo en dos grandes etapas, cada una de las cuales siguió una estrategia metodológica integrada por diferentes fases. En la primera, se empleó la revisión documental de programas de asignatura y documentos oficiales, complementarios a dichos programas, en los cuales se establecen lineamientos para el tratamiento y la evaluación del aprendizaje del tema matemático bajo estudio. En la segunda, mediante un estudio de caso (Sandín, 2003), se analizaron los sistemas de prácticas que dos profesores en un periodo de ocho a nueve sesiones (tiempo dedicado al tema de las ecuaciones lineales) pusieron de manifiesto antes y durante el desarrollo del tema en el salón de clases, así como en sus planeaciones didácticas, materiales de apoyo e instrumentos de evaluación utilizados.

Se realizó una selección de maestros a partir de un proceso de indagación basado en la aplicación de cuestionarios, dirigidos a un conjunto de profesores de álgebra en activo del estado de Sonora, México. Interesaba conocer su formación didáctico-disciplinar, su experiencia docente, sus prácticas e instrumentos habituales de evaluación, así como el impacto de la reforma curricular vigente en su práctica. De este conjunto, se eligieron dos profesores, cada uno de los cuales era perteneciente a uno de los dos subsistemas de bachillerato con mayor cobertura en México (bachillerato general y tecnológico), según la Subsecretaría de Educación Media Superior (SEMS, 2016). Los profesores serán identificados como "profesor A" (asociado al bachillerato tecnológico), y "profesor B" (asociado al bachillerato general).

Las técnicas empleadas para la recolección de datos fueron la entrevista y la observación no participante de la actividad docente de los profesores. Además, se requirieron instrumentos para el registro de la información, tales como cuadros sinópticos, cuestionarios, guion para la entrevista semiestructurada y un formato para el registro de lo observado; éstos fueron diseñados de acuerdo a los constructos teóricos del EOS. La Tabla 1 presenta un resumen de las acciones metodológicas e instrumentos de investigación antes mencionados. 
Tabla 1. Acciones e instrumentos de investigación y su relación con el marco teórico del EOS.

\begin{tabular}{|c|c|c|}
\hline Instrumento & $\begin{array}{l}\text { Propósitos del análisis ontosemiótico de la información } \\
\text { generada por el instrumento }\end{array}$ & $\begin{array}{l}\text { Con este análisis se } \\
\text { construyó }\end{array}$ \\
\hline \multirow{3}{*}{$\begin{array}{l}\text { Cuadro } \\
\text { sinóptico }\end{array}$} & $\begin{array}{l}\text { Identificar el sistema de prácticas promovido para las } \\
\text { ecuaciones lineales desde los programas oficiales de ál- } \\
\text { gebra de dos subsistemas del bachillerato mexicano. }\end{array}$ & \multirow{2}{*}{$\begin{array}{l}\text { Significado } \\
\text { pretendido por el } \\
\text { currículo del } \\
\text { bachillerato } \\
\text { mexicano } \\
\text { (Referencial) }\end{array}$} \\
\hline & $\begin{array}{l}\text { Identificar las prácticas docentes e instrumentos promovi- } \\
\text { dos en los programas de materia y documentos oficiales } \\
\text { (complementarios al programa) de dos subsistemas de } \\
\text { bachillerato, con respecto a la evaluación del aprendizaje } \\
\text { de la noción de ecuación lineal. }\end{array}$ & \\
\hline & $\begin{array}{l}\text { Identificar los sistemas de prácticas pretendidos con res- } \\
\text { pecto a las ecuaciones lineales en los materiales de apo- } \\
\text { yo (libros de texto diseñados) para desarrollar el curso y } \\
\text { en las planeaciones didácticas elaboradas por los sujetos } \\
\text { de estudio. }\end{array}$ & \multirow{2}{*}{$\begin{array}{l}\text { Significado } \\
\text { pretendido por los } \\
\text { profesores }\end{array}$} \\
\hline $\begin{array}{l}\text { Cuestionario y } \\
\text { guion de } \\
\text { entrevista }\end{array}$ & $\begin{array}{l}\text { Identificar a través del discurso de los sujetos de estudio, } \\
\text { las prácticas pretendidas para el abordaje de las ecuacio- } \\
\text { nes lineales y la evaluación de su aprendizaje. Esto previo } \\
\text { a su práctica áulica. }\end{array}$ & \\
\hline \multirow[b]{2}{*}{$\begin{array}{l}\text { Protocolo de } \\
\text { observación }\end{array}$} & $\begin{array}{l}\text { Construir y analizar las trayectorias epistémicas y docen- } \\
\text { tes evidenciadas durante la práctica operativa de los pro- } \\
\text { fesores, a partir del proceso de observación llevado a cabo } \\
\text { en sus aulas. }\end{array}$ & $\begin{array}{l}\text { Significado } \\
\text { implementado }\end{array}$ \\
\hline & $\begin{array}{l}\text { Identificar los sistemas de prácticas puestas de manifiesto } \\
\text { por los profesores al evaluar los aprendizajes de la noción } \\
\text { de ecuación lineal en el bachillerato; colección de tareas } \\
\text { o cuestiones que incluye en las pruebas de evaluación y } \\
\text { pautas de observación de los aprendizajes, así como de } \\
\text { los instrumentos o materiales utilizados. }\end{array}$ & Significado evaluado \\
\hline
\end{tabular}

A continuación, se presenta el análisis de la información generada en cada una de las fases de la investigación, con lo cual se lograron caracterizar los significados institucionales de la noción de ecuación lineal. 


\section{SIGNIFICADOS DE REFERENCIA DE LA ECUACIÓN LINEAL}

En esta sección se describe la caracterización realizada sobre los significados de referencia de la noción de ecuación lineal: a) significado holístico, a partir de un estudio de tipo histórico-documental y; b) significado pretendido por el currículo, a partir del análisis de los programas de estudio y propuestas oficiales.

\subsection{SigNIFICADO HOLÍSTICO DE REFERENCIA DE LA ECUACIÓN LINEAL}

Considerando el interés por determinar cuáles son los significados pretendidos, implementados y efectivamente evaluados por los profesores respecto de la ecuación lineal, fue necesario identificar y establecer (al menos de manera general) cuáles son los significados que ha ido adquiriendo dicha noción a lo largo de la historia; es decir, sus usos y las diversas formas de ver y de entender a la ecuación lineal con el paso del tiempo.

Dichos significados pueden considerarse como un acercamiento al significado global de referencia de dicho objeto matemático. De acuerdo con Pino-Fan, Godino y Font (2011):

El significado global de referencia se define a partir de dos nociones: significado global (también denominado significado holístico u holosignificado, comprende los diferentes significados parciales de un objeto matemático) y significado de referencia (entendido como los sistemas de prácticas que se usan como referencia para elaborar los significados que se pretenden incluir en un proceso de estudio). (p. 147)

La revisión de trabajos de carácter histórico-epistemológico del álgebra, y en particular sobre la noción de ecuación lineal, permitió determinar siete significados parciales como propuesta del significado global de referencia para esta noción matemática. Se presentan de forma sucinta, y tienen el propósito de hacer contrastes entre los significados pretendidos por el currículo, y el pretendido, implementado y evaluado por los profesores, para así poder establecer algunas conclusiones al respecto:

La ecuación lineal como medio para la deducción de valores desconocidos. Esta acepción tiene origen desde las matemáticas babilonias (2000 a.C.-600 a.C.), donde a través de tablillas de barro y con los egipcios (2000 a.C.-1800 a.C.) en sus papiros, quedaron registrados problemas que tratan de la búsqueda de valores 
o cantidades desconocidas sobre situaciones de comercio, herencias, división de propiedades, peso de las cosas, etc., resueltas por el "método de la falsa posición" (Guelli, 1989).

La ecuación lineal como representación de la relación entre magnitudes geométricas. Esta acepción fue considerada por las aportaciones de los griegos (300 a.C.-300 d.C.), quienes desarrollaron estudios y manipulaciones sobre el uso de las ecuaciones lineales en situaciones relacionadas con la geometría y la medición de magnitudes, formas y segmentos (Dalcín y Olave, 2007), así como para construir templos (Boyer, 1968).

La ecuación lineal como relación de proporcionalidad. Esta acepción de ecuación lineal se consideró debido a que, por ejemplo, en el Papiro de Rhind (s. XVII a.C.) se encontraron problemas referentes a intercambios de mercancías o repartos proporcionales. También aparecen estos tipos de problemas en textos chinos del siglo II a.C. (Cullen, 2007), y en textos hindúes (Patwardan et al., 2001).

La ecuación lineal como modelo lineal. Diofanto marcó la modelación de problemas prácticos a través de ecuaciones de difícil deducción, mostrando sus soluciones. Una de sus características fue dar única solución a las ecuaciones, lo que se explica debido a que él buscaba resolver los problemas, no las ecuaciones planteadas. Las soluciones eran números racionales exactos y positivos, a diferencia de los babilónicos que aceptaban aproximaciones irracionales como soluciones a las ecuaciones (Puig, 2006).

La ecuación lineal como la recta en su representación gráfica. Desde la antigüedad, el estudio de las ecuaciones lineales presentó una fuerte relación con situaciones gráficas y geométricas, siendo habitual que a una letra se le designara para representar una magnitud o un objeto, un punto o una recta (Bashmakova y Smirnova, 2000). Los trabajos desarrollados por Viète, Fermat y Descartes, con base en los Elementos de Euclides, presentaron cálculos y construcciones geométricas, así como argumentos involucrando a las ecuaciones lineales (Millán, 2004). Además, según Gulikers y Blom (2001), la idea de línea recta es uno de los conceptos de la geometría que fueron estudiados con mayor profundidad por los griegos.

La ecuación lineal como relación funcional. Esta acepción se consideró ya que Collete (1998) expresa que la representación analítica de la recta es denominada función lineal. Así también Smith y Col (2001) expresan que una función lineal es una cuyo dominio son todos los números reales, cuyo codominio también todos los números reales, y cuya expresión analítica es un polinomio de primer grado. La función lineal se define por la ecuación $f(x)=m x+b$ (o 
$y=m x+b$ ) Ilamada ecuación canónica. Mencionan que una función es una relación entre dos magnitudes o variables numéricas, $x$ e $y$, donde a cada valor de $x$ le corresponde un único valor de $y$.

La ecuación lineal como expresión analítica. Esta última acepción se determinó con base en el comienzo de una llamada "verdadera teoría de ecuaciones", la cual se atribuye generalmente a Viète (Ribnikov, 1987). Posteriormente, Euler llamó al álgebra teoría de los "cálculos con cantidades de distintas clases", donde los conceptos y procedimientos estaban en función de cálculos con números racionales enteros, fracciones ordinarias, raíces cuadradas y cúbicas, progresiones y todo tipo de ecuaciones. Según Smith (2001), una de las formas representativas actuales de una ecuación lineal, es la expresión $a x+b=c$, donde $a, b$ y $c \in \mathbb{R}$, con $a \neq 0$.

Los siete significados parciales expuestos aquí serán retomados en las siguientes secciones, pues permitieron realizar los contrastes pertinentes en los análisis de significados movilizados por los profesores, tal como se mostrará más adelante.

\subsection{SigNiFICADO PRETENDIDO POR EL CURRÍCULO DE BACHILLERATO}

Considerando que uno de los objetivos del estudio fue identificar y caracterizar los sistemas de prácticas promovidos para la noción de ecuación lineal, desde los programas oficiales de álgebra, en dos subsistemas del bachillerato mexicano -tecnológico y general-, se seleccionó al programa oficial del curso como base del significado curricular, ya que según Ibarra (2008), éste contiene la posición institucional de un centro de enseñanza, es el documento que se entrega a un profesor cuando se le asigna la tarea de impartir un curso, y contiene la información de los qué, cómo, en cuánto tiempo, para qué, por qué, etcétera.

Otras fuentes consideradas fueron los documentos oficiales, lineamientos y acuerdos secretariales, que han sido publicados para apuntalar el enfoque de EBC. Dichos documentos fueron seleccionados puesto que, además de ser representativos para los diferentes subsistemas de EMS, en ellos se establecen sugerencias y consideraciones sobre la manera en la que los profesores deben llevar a cabo el proceso de evaluación del aprendizaje en sus aulas, incluyendo el uso de diferentes instrumentos para efectuar esta tarea. 


\subsection{SIGNIFICADO PRETENDIDO POR EL BACHILLERATO TECNOLÓGICO}

La construcción de este significado requirió del análisis de los siguientes documentos: 1) Programa oficial del curso de Álgebra (SEMS, 2013), Unidad 2 “Desarrolla productos notables y viceversa (factorización). Utiliza las propiedades de la igualdad en la resolución de problemas cotidianos que generen ecuaciones de primer grado con una incógnita" (p. 16); 2) ACUERDO número 8/CD/2009 del Comité Directivo del Sistema Nacional de Bachillerato. Orientaciones sobre la evaluación del aprendizaje bajo un enfoque de competencias (SNB, 2009, p. 2); 3) Acuerdo Secretarial 653 (SEP, 2013) por el que se establece el Plan de Estudios del Bachillerato Tecnológico.

El primer significado identificado en el programa oficial del curso de Álgebra para este tipo de bachillerato fue el de ecuación lineal como expresión analítica, pues se declara que el profesor inicie con la presentación del concepto de ecuación, a través de la asociación entre el concepto de igualdad y expresión algebraica, formando con ello el concepto de "igualdad algebraica" con el cual se formaliza el concepto de ecuación. Se prescribe que también se identifiquen los elementos que componen a una ecuación (como el signo igual, incógnita y/o variable, miembros, términos, así como el grado absoluto de la ecuación), para con ellos establecer el concepto de ecuación lineal.

La acepción de ecuación como expresión analítica se mantiene vigente, pues el programa declara que dentro de los sistemas de prácticas que se deben promover está el uso de las propiedades de la igualdad para resolver situaciones-problema en contextos que sean familiares y atractivos para los estudiantes. Se promueve la construcción de modelos lineales que permitan representar la situación-problema y, a su vez, llegar a la solución de ésta; con ello se considera la presencia de la acepción de ecuación lineal como modelo lineal.

Nuevamente la acepción de ecuación lineal como expresión analítica se pone de manifiesto cuando, en relación con las propiedades de la igualdad, también se prescribe que sean utilizadas para promover los despejes entre variables. Para la conclusión del tema se prescribe el planteamiento de situaciones-problema también en contexto intra-matemático, a través de los cuales se promueva la resolución y evaluación de ecuaciones lineales de dos incógnitas, para así terminar con la construcción de gráficas. En ese sentido, se muestran indicios de la promoción de las acepciones de ecuación lineal como relación funcional y el de la recta como su representación gráfica. Para esta última acepción se sugiere la promoción de técnicas para llevar a cabo dichas 
construcciones, partiendo de la ecuación en su forma analítica, después tabular y finalmente graficar, a través de una serie de puntos.

Respecto a la propuesta institucional para la evaluación del aprendizaje de las ecuaciones lineales, fue posible identificar que las prácticas promovidas se centran en verificar la resolución correcta de las situaciones-problema, principalmente en contexto intra-matemático, así como en el cumplimiento y participación dentro de las tareas matemáticas planteadas. Además, se recomienda llevar a cabo tres tipos de evaluación: autoevaluación, coevaluación y heteroevaluación. Los instrumentos que se sugieren para ello son cuestionarios, listas de cotejo, pruebas escritas, guías de observación, cuadros comparativos, matriz de clasificación, registro de competencias, rúbricas y escala de valores.

\subsection{SIGNIFICADO PRETENDIDO POR EL BACHILLERATO GENERAL}

Para la construcción de este significado, se consideraron los siguientes documentos: 1) Programa oficial del curso de Matemáticas 1 (DGB, 2014), Bloque VI: "Resuelves Ecuaciones Lineales I" (p. 33-36); 2) ACUERDO número 8/CD/2009 del Comité Directivo del Sistema Nacional de Bachillerato. Orientaciones sobre la evaluación del aprendizaje bajo un enfoque de competencias (SNB, 2009) y Lineamientos de evaluación del aprendizaje en la educación media superior (DGB, 2011, p. 9-11).

En el programa oficial se identificó la presencia de la acepción de ecuación lineal como expresión analítica, a través de un sistema de prácticas en el que se promueve que el profesor de a conocer de forma breve las características y propiedades de las ecuaciones lineales, así como los conceptos y definiciones asociados a esta noción. Posteriormente se sugiere continuar con la presentación de las diferentes técnicas y/o procedimientos que serán promovidos para el proceso de resolución de las ecuaciones lineales planteadas.

Asimismo, la acepción de ecuación lineal como relación funcional se exhibe cuando se plantea que es necesario que el profesor lleve a cabo acciones que permitan describir e identificar cuál es el comportamiento que tienen las variables y las soluciones obtenidas al resolver problemas sobre ecuaciones y/o funciones lineales en situaciones-problema en contexto intra-matemático. El lenguaje predominante es el algebraico, tanto para llevar a cabo los procedimientos de resolución, como para la comprobación y validación de las soluciones obtenidas. Sin embargo, también se hace explícita la sugerencia de promover 
los lenguajes de tipo numérico, tabular y gráfico para la representación de funciones lineales.

Por otra parte, se sugieren técnicas para la resolución de situaciones-problema que pueden llegar a ser modeladas por medio de una ecuación lineal. El programa de estudios propone algunos contextos extra-matemáticos, tales como mezclas, movimiento rectilíneo uniforme, palancas, entre otros. En ese sentido, la acepción de ecuación lineal como modelo lineal también es promovida. Se prescribe también que el profesor incentive la construcción de gráficas mediante procedimientos tales como análisis de la recta y sus intersecciones con los ejes, pendiente-ordenada al origen y tabulación, con lo cual se identifica el interés en que aparezca el significado de ecuación lineal como la recta en su representación gráfica, usándola además para comprobar e interpretar las soluciones obtenidas.

También se identificó la presencia de la acepción de ecuación lineal como representación de la relación entre magnitudes geométricas, ya que otro de los aspectos que se plantean en el currículo, pone de manifiesto que el sistema de prácticas del profesor debe estar orientado a la construcción e interpretación de la ecuación y/o función lineal, mediante el desarrollo de procedimientos aritméticos, algebraicos, geométricos y variacionales. Para ello, el currículo sugiere que también se promueva la comprensión y análisis de situaciones-problema en contexto extra-matemático, que estén asociadas con el entorno del estudiante.

En cuanto a la propuesta institucional del bachillerato general para la evaluación del aprendizaje de las ecuaciones lineales, se indica el planteamiento de situaciones-problema en las que se valore el progreso del estudiante a través de prácticas que pongan en juego los diferentes objetos matemáticos primarios, así como el desarrollo de competencias tanto genéricas como disciplinares. Algunas de las acciones o procesos que son promovidos y susceptibles de ser evaluados, son la resolución de problemas, la interpretación y validación de soluciones obtenidas, la argumentación de los procedimientos realizados y soluciones obtenidas, el manejo y diseño de tablas y gráficos, la modelación de situaciones-problema que involucren el uso de ecuaciones lineales y el manejo de diferentes lenguajes y sus diversos tipos de representación. Los instrumentos sugeridos para ello son: listas de cotejo, escalas de clasificación, problemarios resueltos y portafolios de evidencias. 


\section{SIGNIFICADOS DE LA ECUACIÓN LINEAL PRETENDIDOS POR LOS PROFESORES}

El significado pretendido por los profesores, de acuerdo con el EOS, se conforma por el sistema de prácticas operativas y discursivas que ponen en juego en la planificación de sus clases, tomando como base un significado de referencia (en este caso el pretendido por el currículo oficial). Para los fines de esta investigación, fue necesario caracterizar el significado pretendido por cada profesor, ya que las situaciones y los contextos en los cuales planifican sus clases son distintos. A continuación se describen los análisis sobre los significados pretendidos por los profesores A (bachillerato tecnológico) y B (bachillerato general). Cabe señalar que además de sus planificaciones, para complementar dicha caracterización se diseñaron e implementaron un cuestionario y una entrevista semiestructurada.

\subsection{SIGNIFICADOS PRETENDIDOS POR EL PROFESOR A}

En los hechos el profesor A no desarrolló una planeación didáctica del curso, expresando que se guía totalmente por lo que el libro de texto establece, ya que acostumbra abordar las situaciones problema tal y como se sugieren, asignando el mismo tiempo a cada unidad temática independientemente de los contenidos que se aborden. Además, argumentó la necesidad de trabajar así para que los estudiantes se preparen para el examen final, el cual es centralizado (un único examen aplicado a todos los estudiantes de la institución). No obstante, manifestó la necesidad de aplicar un examen al término de cada tema, esto con el fin de valorar el aprendizaje de sus estudiantes y poder cumplir con el requerimiento institucional (entrega de calificaciones).

A partir del análisis ontosemiótico se identificó un interés prioritario en promover el significado de ecuación lineal como expresión analítica, pues a través de esta acepción se gestionan los elementos que componen a la ecuación lineal, así como las técnicas de resolución dentro del mismo lenguaje algebraico. Otro de los significados presentes fue el de la ecuación como modelo lineal, al detectar que la mayoría de los problemas propuestos en el texto ya presentan el modelo algebraico con el cual es obtenida su solución.

El profesor A señaló que promueve la construcción de gráficas, partiendo de expresiones analíticas, es decir, ecuaciones lineales de dos variables donde 
se recurre a la graficación a través de una serie de puntos previamente calculados y colocados en un registro tabular. Este hecho confirma que se abordan de una manera muy incipiente las acepciones de la ecuación lineal como la recta en su representación gráfica y como relación funcional. Asimismo, se advierte que tiene planificado que, después de promover los aspectos gráficos relacionados con la ecuación lineal de dos variables, se retomen nuevamente las situaciones-problema en contexto intra-matemático para reafirmar las técnicas de resolución de las ecuaciones lineales en su expresión analítica.

Complementario a esto, se encontró que dentro del libro de texto se establece una evaluación del aprendizaje a través de rúbricas que se centran en ponderar el interés y la actitud del estudiante, la cantidad de ejercicios resueltos correctamente y el nivel de cumplimiento en la entrega de tareas. A pesar de que se contempla una evaluación diagnóstica y una ficha de autoevaluación que plantea la reproducción de algoritmos, todos estos recursos se alejan de una evaluación de tipo formativa. No obstante, a partir de sus respuestas en la entrevista, se logró identificar que concibe a la evaluación del aprendizaje como una acción temporal, al final del proceso de enseñanza; lo cual le marca la pauta para valorar el dominio de las habilidades y conocimientos (significado) que los estudiantes tienen del objeto. Manifestó que esto es llevado a cabo a través de ciertos registros, pero sobre todo por medio del examen donde se solicita la reproducción de procedimientos y definiciones.

\subsection{SIGNIFICADOS PRETENDIDOS POR EL PROFESOR B}

Para la determinación del significado pretendido por el profesor, se analizó el formato de planeación que proporcionó, con el fragmento correspondiente al tema de las ecuaciones lineales. Una vez realizado el análisis ontosemiótico, se identificó la promoción del significado de ecuación lineal como expresión analítica, debido al interés mostrado por la exploración de técnicas para su solución y la formalización de su definición. Posteriormente surgió la acepción de ecuación lineal como modelo lineal, pues a través de ella se planificó el planteamiento de situaciones-problema que pueden ser modeladas y resueltas a través de una ecuación lineal, independientemente del tipo de coeficientes.

Además, se logró identificar la presencia de situaciones-problema en contextos extra-matemáticos, a través de los cuales se construye el significado de ecuación lineal como relación de proporcionalidad, pues dichas situaciones se planificaron 
con el fin de impulsar en los estudiantes la identificación de la relación existente entre la ecuación y la función lineal.

En relación con la evaluación del aprendizaje, los instrumentos que se sugieren son listas de cotejo, problemarios resueltos y portafolios de evidencias; se pretenden evaluaciones diagnóstica, sumativa, formativa, autoevaluación y coevaluación. En su discurso el profesor externó que se enfoca en la "identificación de logros de aprendizaje y detección de dificultades presentes al trabajar con el objeto", enfocándose en "encaminar a sus estudiantes hacia la construcción de un significado más completo del objeto a través de la validación de sus acciones y retroalimentación constante".

\section{SIGNIFICADOS IMPLEMENTADOS POR LOS PROFESORES}

De acuerdo con el EOS, el significado implementado refiere al sistema de prácticas efectivamente manifestadas por el docente, durante un proceso de instrucción (Godino et al., 2009); esto es, lo que en realidad hizo y dijo el profesor en el aula al abordar un tema matemático, en su carácter de representante de la institución.

\subsection{SIGNIFICADOS IMPLEMENTADOS POR EL PROFESOR A}

El profesor A abordó el tema de las ecuaciones lineales en un periodo de 9 sesiones de 45 minutos cada una. Sin embargo, el tiempo efectivo fluctuaba entre 30 y 42 minutos por sesión, situación que lo obligó a trabajar a "marchas forzadas" para poder cubrir lo que el libro de texto establece.

Inicialmente fue posible identificar su interés por resaltar la importancia del tema, y con ello mostrar la aplicabilidad de las técnicas algebraicas y de algunos conceptos involucrados, lo cual dio paso a la acepción de ecuación lineal como expresión analítica. Dado que mencionó constantemente que las ecuaciones lineales tenían diversas aplicaciones en la vida diaria, el profesor expresó a los estudiantes que resolverían durante las sesiones algunas situaciones que les resultarían familiares. En ese sentido, el planteamiento de situaciones-problema en contextos extra-matemáticos sencillos como el manejo de dinero, cálculo de edades, ventas, etc., permitió introducir la acepción de ecuación 
lineal como modelo lineal en la resolución de situaciones-problema en contextos intra-matemáticos.

La ecuación lineal como relación funcional y como la recta en su representación gráfica, aparecieron vagamente en una de las situaciones-problema sugeridas por el texto y retomada por el profesor. En dicha situación se llevaron a cabo los procesos necesarios para su resolución y no se hicieron explícitos en ningún momento los conceptos de función, pendiente, variables, etc., así que los estudiantes se quedaron con la idea de que se trataba únicamente de una ecuación lineal con dos incógnitas, la cual les permitía obtener pares ordenados que podían ser localizados en el plano cartesiano. Al final de las sesiones y dado que no se llevaba un control de las clases, se retomó la acepción de ecuación lineal como expresión analítica para resolver problemas sobre despejes de variables, y la acepción de ecuación lineal como modelo lineal para resolver un problema extra-matemático.

\subsection{SIGNIFICADOS IMPLEMENTADOS POR EL PROFESOR B}

El profesor B desarrolló el tema de las ecuaciones lineales en un periodo de 8 sesiones de 50 minutos cada una. Se podría decir que abordó el tema en el tiempo programado dentro de la planeación del bachillerato general. Asimismo, se dio a la tarea de llevar a cabo algunas acciones que le permitieran identificar los conocimientos previos de sus estudiantes, incorporando además algunos temas matemáticos que no estaban contemplados en su significado de referencia (programa oficial de materia).

El profesor promovió al inicio las acepciones de ecuación lineal como medio para la deducción de valores desconocidos y como expresión analítica. Recurrió, aunque de manera muy pobre, a la acepción de ecuación lineal como representación de la relación entre magnitudes geométricas. Durante el resto de las sesiones, optó por promover inicialmente la acepción de ecuación lineal como expresión analítica con el fin de formalizar y demostrar, a través de su discurso, los elementos que componen una ecuación. Posteriormente se llevó a cabo una presentación de conceptos y antecedentes históricos del tema, concluyendo con la acepción de ecuación lineal como modelo lineal para resolver el problema del misterio de la edad de Diofanto. A partir de ese momento la acepción como modelo lineal recobró fuerza en el resto de las situaciones-problema planteadas. 
Además de promover la modelación, se plantearon situaciones-problemas asociadas con el tema de proporcionalidad directa. En ese sentido se resolvieron problemas que permitieron formalizar algunos conceptos como constante de proporcionalidad y variable; se insistió en el uso de los lenguajes tabular y gráfico, y con ello se institucionalizaron los conceptos de función, variable dependiente e independiente, así como algunos aspectos gráficos como recta y pendiente; además de impulsar procesos de generalización a través de una expresión algebraica, la cual era llamada "fórmula".

\section{SIGNIFICADOS EVALUADOS POR LOS PROFESORES}

En el EOS, el significado evaluado alude al subsistema de prácticas que utiliza el docente para evaluar los aprendizajes matemáticos durante el proceso de instrucción (Godino et al., 2009). En ese sentido, la tarea consistió en identificar y caracterizar las prácticas puestas en juego por los dos profesores, al evaluar los aprendizajes sobre la noción de ecuación lineal, analizando también los instrumentos utilizados durante la implementación de dichas prácticas evaluativas, así como los significados evaluados de la noción de ecuación lineal.

\subsection{PRÁcticas, INSTRUMENTOS Y SIGNIFICAdOS EVAlUadOS POR EL PROFESOR A}

Inicialmente fue posible identificar su interés en dar cumplimiento tanto a las propuestas sugeridas y pretendidas por su institución, es decir, aplicar el examen diagnóstico y la ficha de autoevaluación, ambos propuestos por el libro de texto; así como preparar a los estudiantes para el día de la aplicación del examen, resolviendo y estudiando las situaciones-problema planteadas por el mismo texto. Durante las sesiones, las prácticas evaluativas del profesor A se centraban en realizar constantes cuestionamientos a los estudiantes sobre las propuestas de procedimientos de resolución de una ecuación, las soluciones obtenidas y sobre los argumentos necesarios para justificar tales resultados. Frecuentemente se cuestionó a los estudiantes sobre conceptos y procedimientos que habían estudiado con anterioridad, todo esto con el fin de verificar si habían sido "comprendidos".

Durante las sesiones de clase, el profesor identificó dificultades manifestadas por los estudiantes al abordar las situaciones-problema planteadas. Casi siempre, 
cuando se presentaba una situación de ese tipo, el profesor iniciaba una discusión grupal buscando, mediante interrogantes, evidenciar los errores, y con ello aclarar las dudas que llegaban a externar los estudiantes. No siempre los resultados de las discusiones fueron favorables, lo cual ocasionaba que el profesor se desesperara y terminara resolviendo los problemas, construyendo los modelos o diciendo él mismo la solución, argumentando siempre que "no era nada complejo" y asignando tareas para promover su reproducción.

A pesar de que implementó el examen diagnóstico y llevaba un registro diario sobre las conductas de los estudiantes, el profesor tenía claro que la evaluación se realizaría al final del proceso de instrucción mediante la aplicación de un examen final (evaluación sumativa) diseñado por él mismo, tomando como base las situaciones-problema del texto. De acuerdo con las prácticas implementadas y promovidas por este profesor, es posible concluir que el significado evaluado sobre la noción de ecuación lineal, se centró en el planteamiento de situaciones relacionadas con las acepciones de expresión analítica y modelo lineal, así como con el de relación funcional.

Fue posible distinguir que dentro de las prácticas del profesor $\mathrm{A}$, no se tenía el propósito de llevar a cabo acciones evaluativas a lo largo de la instrucción, debido a que concibe a la evaluación como una acción que se realiza al final del proceso de enseñanza. Por tal motivo, se mantuvo ausente la retroalimentación y la comunicación de los resultados, avances y/o logros obtenidos. El papel de los estudiantes consistió en demostrar, a través de las pruebas escritas, los conocimientos adquiridos sobre la ecuación lineal.

\section{Correspondencia entre los significados del profesor $A$}

Al determinar los significados curriculares se concluyó que, a partir de lo planteado por el programa del bachillerato tecnológico, los significados de la ecuación lineal propuestos se centraron en las acepciones de expresión analítica, modelo lineal y la recta en su representación gráfica. Posteriormente se concluyó que los significados de la ecuación lineal pretendidos por el profesor A fueron los de modelo lineal y expresión analítica; en apartados anteriores se presentó que el significado de la ecuación lineal implementado por el profesor A consistió esencialmente en dos acepciones: expresión analítica y modelo lineal. Finalmente, en esta sección se describió que el significado de la ecuación lineal evaluado por el profesor A se centró en esas dos mismas acepciones: expresión analítica 
y modelo lineal, aunque también se tuvo la presencia de la ecuación como relación funcional. Asimismo, se destacó la presencia de pequeños esbozos sobre el significado de ecuación lineal como la recta en su representación gráfica tanto en lo pretendido como en lo implementado.

La Tabla 2 que se muestra a continuación, resume lo descrito.

Tabla 2. Correspondencia entre los significados de la ecuación lineal por el profesor A

\begin{tabular}{|c|c|c|c|c|}
\hline $\begin{array}{l}\text { Significados parciales de la } \\
\text { noción de ecuación lineal }\end{array}$ & $\begin{array}{c}\text { Significado } \\
\text { Pretendido por } \\
\text { el currículo }\end{array}$ & $\begin{array}{c}\text { Significado } \\
\text { Pretendido por } \\
\text { el profesor }\end{array}$ & $\begin{array}{c}\text { Significado } \\
\text { Implementado } \\
\text { por el profesor }\end{array}$ & $\begin{array}{l}\text { Significado } \\
\text { Evaluado por } \\
\text { el profesor }\end{array}$ \\
\hline $\begin{array}{l}\text { 1. Como medio para la } \\
\text { deducción de valores } \\
\text { desconocidos }\end{array}$ & & & & \\
\hline $\begin{array}{l}\text { 2. Como representación de } \\
\text { relación entre magnitudes } \\
\text { geométricas }\end{array}$ & & & & \\
\hline $\begin{array}{l}\text { 3. Como relación de } \\
\text { proporcionalidad }\end{array}$ & & & & \\
\hline 4. Como modelo lineal & & & & \\
\hline $\begin{array}{l}\text { 5. Como la recta en su } \\
\text { representación gráfica }\end{array}$ & & & & \\
\hline 6. Como relación funcional & $x$ & x & $?$ & \\
\hline 7. Como expresión analítica & & & & \\
\hline
\end{tabular}

\begin{tabular}{|c|c|}
\hline \multicolumn{2}{|c|}{ Simbología } \\
\hline$\circlearrowright$ & Promovido \\
\hline$!$ & $\begin{array}{c}\text { Promovido } \\
\text { parcialmente }\end{array}$ \\
\hline$\times$ & No promovido \\
\hline
\end{tabular}

\subsection{PRÁCTICAS, INSTRUMENTOS Y SIGNIFICADOS EVALUADOS POR EL PROFESOR B}

Identificamos que las prácticas de evaluación manifestadas por el profesor B fueron diversas y con diferentes fines. En la etapa inicial del proceso de instrucción, el profesor valoró los aprendizajes previos de los estudiantes, movilizando objetos matemáticos a través de situaciones-problema en contextos intra-matemáticos, calculando valores desconocidos, resolviendo problemas en contextos geométricos y situaciones-problemas sobre ecuaciones lineales como expresiones analíticas.

Durante el desarrollo del tema evaluó constantemente el desempeño de los estudiantes mediante cuestionamientos dirigidos a constatar su nivel de argumentación, así como la seguridad para verbalizar los procedimientos de resolución de los problemas planteados. A través de estas situaciones-problema, el 
profesor cuestionó a los estudiantes y llevó a cabo procesos de retroalimentación y discusiones grupales. Las situaciones-problema estuvieron mayoritariamente planteadas con el propósito de identificar los elementos de una ecuación lineal y fomentar técnicas de resolución algebraica, modelar enunciados verbales, situaciones de comportamiento lineal (proporcionalidad directa) o que son modelados a través de una función lineal en sus respectivas representaciones tabular y gráfica.

La observación constante del trabajo individual y por equipos, permitió al profesor llevar a cabo una evaluación con la que, al final del proceso de instrucción, identificó a los estudiantes que necesitaban asesorías extra-clase. Complementó su evaluación a través de la autoevaluación y coevaluación de tipo académica y actitudinal. Finalmente, se aplicó un examen escrito, el cual se estructuró con problemas sobre ecuaciones lineales, situaciones-problema en contextos intra y extra-matemáticos relacionados con proporcionalidad y modelados por funciones lineales.

Se concluye entonces que, el significado evaluado del profesor B se enfocó prioritariamente en 5 de los significados parciales de la noción de ecuación lineal (aunque se reflejó presencia de los 7): la ecuación lineal como medio para la deducción de valores desconocidos, relación de proporcionalidad, modelo lineal, relación funcional y expresión analítica.

Fue posible distinguir que el profesor $\mathrm{B}$, desde su planificación, contempla varias acciones para llevar a cabo la evaluación del aprendizaje de sus estudiantes; por ejemplo, en sus objetivos declaró la implementación de los tres tipos de evaluación; diagnóstica, formativa y sumativa. En el caso de la evaluación diagnóstica, Casanova (2007) la define como la evaluación inicial que tiene por objetivo proporcionar información acerca de los conocimientos y las habilidades previas del sujeto. En ese sentido, el profesor implementó estos tres tipos de evaluación con la intención de comunicar al estudiante su grado de avance y resultados obtenidos. Además de esto, constantemente el profesor recurrió a la retroalimentación, a través de discusiones grupales guiadas. El papel de los estudiantes durante el proceso de estudio consistió en participar activamente en cada una de las tareas. 


\section{Correspondencia entre los significados del profesor $B$}

En la sección del significado curricular, se concluyó que el programa del bachillerato general promovía 5 de los 7 significados parciales de la ecuación lineal: representación de la relación entre magnitudes geométricas, modelo lineal, la recta en su representación gráfica, como relación funcional y expresión analítica. En cuanto a los significados pretendidos por el profesor B, encontramos que este profesor movilizó a la ecuación lineal en sus acepciones de expresión analítica, modelo lineal, relación de proporcionalidad, relación funcional y la recta en su representación gráfica. De estos cinco significados, en el discurso del profesor solo se manifestaron cuatro de ellos, es decir, no se hizo notorio el significado de ecuación como la recta en su representación gráfica. No obstante, se percibieron indicios sobre el significado de ecuación lineal como medio para la deducción de valores desconocidos, el cual fue parcialmente manifestado por el profesor dentro de su planificación.

En la sección de significados implementados se mencionó que el profesor priorizó cuatro significados parciales para la noción de ecuación lineal: relación de proporcionalidad, modelo lineal, como relación funcional y expresión analítica, aunque los otros tres fueron gestionados parcialmente. Se expuso también que el significado evaluado por el profesor B se enfocó prioritariamente en cinco significados parciales: medio para la deducción de valores desconocidos, relación de proporcionalidad, modelo lineal, como relación funcional y expresión analítica. La Tabla 3 resume la correspondencia entre los significados descritos para el profesor B.

Resalta que, en el caso del profesor B, se presentó una alta correspondencia entre los significados implementados y los efectivamente evaluados. Además, se puede concluir que consideró, evidentemente, los significados pretendidos por el currículo para llevar a cabo su planeación (significado pretendido por él mismo). Algo interesante después de estos contrastes fue haber identificado que tanto el significado de ecuación lineal como modelo lineal, relación funcional y como expresión analítica, fueron los únicos tres significados que se manifestaron tanto en el significado curricular como en los significados del profesor. Esto hace inferir que tanto el currículo, como la institución, influyeron en la selección de significados que se manifestaron en sus prácticas. 
Tabla 3. Correspondencia entre los significados de la ecuación lineal por el profesor B

\begin{tabular}{|c|c|c|c|c|c|c|}
\hline $\begin{array}{l}\text { Significados parciales de la } \\
\text { noción de ecuación lineal }\end{array}$ & $\begin{array}{l}\text { Significado } \\
\text { Pretendido por } \\
\text { el currículo }\end{array}$ & $\begin{array}{l}\text { Significado } \\
\text { Pretendido por } \\
\text { el profesor }\end{array}$ & $\begin{array}{l}\text { Significado } \\
\text { Implementado } \\
\text { por el profesor }\end{array}$ & $\begin{array}{l}\text { Significado } \\
\text { Evaluado por } \\
\text { el profesor }\end{array}$ & & \\
\hline $\begin{array}{l}\text { 1. Como medio para la } \\
\text { deducción de valores } \\
\text { desconocidos }\end{array}$ & & & & & & \\
\hline $\begin{array}{l}\text { 2. Como representación de } \\
\text { relación entre magnitudes } \\
\text { geométricas }\end{array}$ & & & & & & \\
\hline $\begin{array}{l}\text { 3. Como relación de } \\
\text { proporcionalidad }\end{array}$ & & & & & & \\
\hline 4. Como modelo lineal & & & & & & \\
\hline 5. Como la recta en su & & & & & & imbología \\
\hline representación gráfica & & & & & 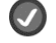 & Promovido \\
\hline 6. Como relación funcional & & & & & ? & $\begin{array}{c}\text { Promovido } \\
\text { parcialmente }\end{array}$ \\
\hline 7. Como expresión analítica & & & & & x & No promovido \\
\hline
\end{tabular}

\section{REFLEXIONES FINALES}

El desarrollo de este estudio permitió recolectar evidencias sobre la manera en la cual se concibe y se lleva a cabo la evaluación del aprendizaje por profesores de matemáticas. En este reporte, el centro estuvo puesto sobre la evaluación de la noción de ecuación lineal, lo cual permitió identificar aquellos factores que jugaron un papel determinante en las prácticas de los profesores, desde su planeación hasta su ejecución (implementación y evaluación del tema).

La reconstrucción del significado holístico de referencia (propuesta de los siete significados parciales del objeto matemático), permitió contar con un referente epistémico sobre la noción de ecuación lineal con el cual fue posible caracterizar y relacionar con mayor precisión tanto las prácticas como los significados puestos de manifiesto por los profesores en su labor áulica.

Considerando que este significado fue utilizado para llevar a cabo los contrastes respecto al significado curricular y los significados pretendidos, implementados y evaluados por los profesores, se pudo observar, en el caso del profesor A, que tanto el significado curricular como su propio significado pretendido fueron muy similares, situación que ocasionó que los diversos significados promovidos de la noción matemática no se correspondieran con el significado holístico de referencia. 
Dicha situación jugó un papel fundamental en sus prácticas, bajo el argumento imperativo de acatar lo que el currículo y el texto le establecen, cumpliendo con ello normativas institucionales relacionadas con el calendario escolar y con la preparación necesaria de los estudiantes para abordar el examen final del tema matemático en estudio, aspectos imprescindibles para emitir un juicio sobre su aprendizaje, el cual concretó vía una calificación.

Con respecto a la evaluación, el profesor A sólo consideró dos de los siete significados parciales. Tanto en su práctica operativa como discursiva, concibió la evaluación del aprendizaje como una acción temporal y no como un proceso permanente. En ese sentido, recurrió a evaluación tanto diagnóstica como sumativa, pues las acciones clasificadas como evaluativas dentro de su trayectoria docente, constataron su interés por verificar que los estudiantes adquirieran las técnicas y las habilidades algorítmicas necesarias para resolver un problema, así como para verbalizar y justificar las soluciones obtenidas o la memorización de algunos conceptos. En la práctica del profesor no fue posible identificar que llevara a cabo procesos de retroalimentación o cambios de estrategia para enfrentar una dificultad o resolver conflictos. A pesar de que se desarrollaban discusiones grupales y cuestionamientos, el profesor optaba por la institucionalización. Aunque en el currículo y en el libro de texto se proponen diferentes instrumentos de evaluación, su uso fue muy pobre. Se destaca que el profesor externó que el examen escrito representaba $20 \%$ de la calificación obtenida en la evaluación sumativa. Argumentó que esto es así, debido a que "ya se sabe que los estudiantes fallan", pero que de alguna u otra manera se debe cumplir con el requisito institucional de aplicar una evaluación de este tipo.

En ese sentido, la práctica del profesor A careció de espacios a través de los cuales pudiera dar a conocer resultados, áreas de oportunidad o de mejora a sus estudiantes; no los hizo partícipes de alguna retroalimentación en las tareas asignadas o exámenes aplicados; solamente les hacía saber "que las cosas no andaban bien", generando un ambiente de frustración e incertidumbre en ellos.

En lo que al profesor B respecta, como se pudo observar, activó una mayor riqueza de significados de la noción matemática, siendo interesante haber identificado que presentó en su planificación la inclusión de significados (pretendidos) que no se encontraban declarados en el currículo; un ejemplo fue el significado de la ecuación lineal como relación de proporcionalidad, considerada en el proceso de enseñanza-aprendizaje de dicha noción matemática.

Respecto a la evaluación del aprendizaje, un aspecto interesante fue que el profesor B dio a conocer, desde el inicio del tema, los criterios y factores 
considerados en la evaluación sumativa, tal y como se lo indicaba su planificación. Mediante la evaluación diagnóstica, el profesor logró procesos de retroalimentación e institucionalización, respecto de los diferentes objetos matemáticos intervinientes durante el estudio de la noción de ecuación lineal. Durante el proceso de instrucción, además de desarrollar plenarias y discusiones grupales, así como trabajo individual y en equipo, también complementó la evaluación a través de una autoevaluación y coevaluación de tipo académica y actitudinal, aunque su trayectoria docente permitió evidenciar que no en todas las sesiones desarrolló acciones con fines puramente evaluativos.

Si bien, durante el desarrollo del tema el profesor manifestó interés en llevar a cabo diferentes acciones relacionadas con la evaluación del aprendizaje y la valoración del progreso de sus estudiantes, se identificó que al momento de implementar la prueba escrita al final del proceso de instrucción, en su estructura priorizó asuntos conceptuales y procedimentales, abordando problemáticas en contextos puramente matemáticos. Aun así, fue muy destacable que en su discurso externara un significado amplio respecto a la evaluación del aprendizaje, concibiéndola como un proceso permanente, en donde sus prácticas no se limitan sólo a emitir juicios o realizar mediciones.

En términos generales el profesor implementó los diversos tipos de evaluación que establecen los lineamientos del modelo curricular y el EBC en México, además de que mostró interés por comunicar a sus estudiantes su grado de avance y resultados obtenidos, así como de fomentar la activa participación durante el proceso de estudio o en cada tarea asignada.

Con lo descrito hasta aquí, se logra evidenciar lo complejo que resulta para los profesores realizar un proceso de evaluación de tipo formativa, que se desarrolle de manera permanente y efectiva, de tal forma que los estudiantes puedan no sólo conocer sus errores o dificultades, sino que a partir de ello puedan reconocer espacios de oportunidad para poder llegar a construir un significado rico y sólido sobre la noción matemática que se esté estudiando. También es posible concluir que, a pesar de que se promueve actualmente en el bachillerato mexicano un modelo de EBC con iniciativas para modificar el significado de evaluación en los profesores, estos procesos de cambio requieren mucho tiempo. Una muestra de ello es que, aunque se dispone de diferentes instrumentos, el examen prevalece como protagonista, siendo aplicado al final del proceso de instrucción y utilizado para emitir juicios sobre el "aprendizaje matemático" a partir de la reproducción de procedimientos y conceptos estudiados. 
Los factores que se identificaron como determinantes en las prácticas de evaluación de los profesores fueron: el significado pretendido por el currículo nacional; las normativas institucionales; sus propias creencias y concepciones acerca de la evaluación; el contexto en el que se desarrolla la clase y sus significados personales (conocimientos didáctico-matemáticos) sobre la noción bajo estudio.

Finalmente se señala que una línea de investigación susceptible de desprenderse de este trabajo es el análisis de los significados declarados y logrados por los estudiantes ante las prácticas de los profesores, entre algunos otros aspectos relacionados con la evaluación del aprendizaje en matemáticas.

\section{AGRADECIMIENTOS}

Este trabajo ha sido desarrollado en el marco de los proyectos de investigación del equipo de trabajo del Programa de la Maestría en Ciencias con Especialidad en Matemática Educativa de la Universidad de Sonora, México, en colaboración con un profesor investigador del programa de Educación Matemática de la Universidad de Los Lagos, Chile.

\section{REFERENCIAS}

Álvarez, R. y Blanco, L. J. (2014). Sobre la evaluación en matemáticas en secundaria. Suma, 76, p. 47-54.

Bashmakova, I. y Smirnova, G. (2000). The Beginnings and Evolution of Algebra. The Mathematical Association of America.

Becerra, R. y Moya, A. (2008). Una perspectiva crítica de la evaluación en matemática en la Educación Media Superior. Sapiens. Revista de Investigación, 9(1), 35-69.

Boyer, C. (1968). A history of mathematics. Wiley International Edition. John Wiley \& Sons, Inc. Library of Congress catalog card number 16506. Brooklyn College, pp. 9-583.

Calderón, R. M y Deiros, B. (2003). Evaluación del aprendizaje de las matemáticas. En J. Delgado Rubí (Ed.), Acta Latinoamericana de Matemática Educativa (Vol. 16. 329333). Comité Latinoamericano de Matemática Educativa.

Casanova, M. A. (2007). Manual de evaluación educativa. 9a Ed. Editorial la Muralla, S. A. Collete, J. (1998). Historia de las matemáticas, vol. 2, Siglo XXI Editores. 
Cullen, C. (2007). The Suàn shù sh, 'Writings on reckoning': Rewriting the history of early Chinese mathematics in the light of an excavated manuscript, Historia Mathematica, 34(1), 10-44.

Dalcín, M. y Olave, M. (2007) Ecuaciones de primer grado: su historia. En C. Crespo (Ed.), Acta Latinoamericana de Matemática Educativa (Vol. 20, pp. 156-161), Comité Latinoamericano de Matemática Educativa.

Dirección General de Bachillerato (2011). Lineamientos de evaluación del aprendizaje en la educación media superior. SEP, DOF 01/07/2011.

Dirección General de Bachillerato (2014). Matemáticas 1. Serie: Programas de estudio. Sistema Nacional de Bachillerato, SEP.

Dolores, C. y García, J. (2016). Concepciones de profesores de matemáticas sobre la evaluación y las competencias. Números, Revista didáctica de las matemáticas, 92(1), 71-92.

Dolores, C. y García, J. (2017). Concepciones de profesores de matemáticas acerca de la evaluación vistas a la luz de la reforma educativa actual en México. Revista Paradigma, XXXVIII(1), 186-210.

Godino, J. D. Batanero, C. y Font, V. (2007). The onto-semiotic approach to research in mathematics education. ZDM. The International Journal on Mathematics Education, 39(1-2), 127-135.

Godino, J. D. y Batanero, C. (1994). Significado institucional y personal de los objetos matemáticos. Recherches en Didactique des Mathématiques, 14(3), 325-355.

Godino, J. D., Contreras, A. y Font, V. (2006). Análisis de procesos de instrucción basado en el enfoque ontológico-semiótico de la cognición matemática. Recherches en Didactique des Mathematiques, 26(1), 39-88.

Goñi, J. M. (2008). La evaluación de las competencias determinará el currículo de matemáticas. En J. M. Goñi (Ed.), 32-2 ideas clave. El desarrollo de la competencia matemática (pp. 167-185). GRAO.

Guelli, O. (1989). A regra da falsa posição. Revista do Profesor de Matemática, 15, 18-22.

Gulikers, I. y Blom, K. (2001). 'A historical angle', a survey of recent literature on the use and value of history in geometrical education. Educational Studies in Mathematics, 47(2), 223-258.

Harlen, W. (2012). The role of assessment in developing motivation for learning. En J. Gardner (Ed.), Assessment and Learning (pp. 171-183). Sage.

Hernández, K. (2013). Representaciones sociales sobre la evaluación en matemáticas en el nivel superior. (Tesis de Maestría no publicada) CICATA-IPN.

Ibarra, S. (2008). La transposición didáctica del álgebra en las ingenierías. El caso de los sistemas de ecuaciones lineales. (Tesis de Maestría no publicada) CICATA-IPN.

Millán, A. (2004). Euclides. La fuerza del razonamiento matemático. Nivola. 
Moreno, (2012). Evaluación cualitativa del aprendizaje: enfoques y tendencias. Revista de la Educación Superior, ANUIES, XXXIII 3(131), 93-110.

Moya, A. (2001). Reflexiones sobre la teoría y práctica de la evaluación en educación matemática. Boletín de Investigación, UPEL, J. M. Siso Martínez, vol. (1).

National Council of Teachers of Mathematics. (1989). Curriculum and evaluation standards for school mathematics. NCTM.

Patwardan, K., Naimpally, S. y Singh, A. (2001). Lilavati of Bhaskaracarya. A treatise of mathematics of vedic tradition. Motilal Banarsidass Publishers.

Pino-Fan, L., Godino, J. D., y Font, V. (2011). Faceta epistémica del conocimiento didáctico matemático sobre la derivada [The epistemic facet of mathematical and didactic knowledge about the derivative]. Educação Matemática Pesquisa, 13(1), 141-178.

Pino-Fan, L., Godino, J. D. y Font, V. (2015). Una propuesta para el análisis de las prácticas matemáticas de futuros profesores sobre derivadas. BOLEMA, 29(51), 60-89.

Puig, L. (2006). La resolución de problemas en la historia de las matemáticas En Aymerich, José V. y Macario, Sergio (Eds.) Matemáticas para el siglo XXI (pp. 39-57) Publicacions de la Universitat Jaume I.

Ribnikov, K. (1987): Historia de las Matemáticas. Editorial Mir. http://thales.cica.es/rd/Recursos/Matematicas/14/historia.html

Sandín, E. (2003). Investigación cualitativa en educación. Fundamentos y tradiciones. Capítulo 7. Mc Graw and Hill Interamericana.

Secretaría de Educación Pública. (2009). Acuerdo secretarial núm. 8: Orientaciones sobre la evaluación del aprendizaje bajo un enfoque de competencias. Comité Directivo del Sistema Nacional de Bachillerato, DOF 8/CD/2009.

Secretaría de Educación Pública. (2013). Acuerdo secretarial núm. 653: Por el que se establece el Plan de Estudios del Bachillerato Tecnológico. DOF.

Shepard, L. A. (2000). The role of assessment in a learning culture. Educational Researcher, 29(7), 4-14.

Smith, Stanley y Col. (2001). Álgebra. Addison-Wesley Iberoamericana.

Stiggins, R. J. (2001). Student-involved classroom assessment, $3^{\underline{a}}$ ed. Prentice Hall.

Subsecretaría de Educación Media Superior (2016). Estadísticas e indicadores del Sistema

Educativo Nacional Mexicano. Dirección General de Planeación, Programación y Estadística Educativa (1) SEP.

Vincenzi de, A. y Angelis de P. (2008). La evaluación de los aprendizajes de los alumnos. Orientaciones para el diseño de instrumentos de evaluación. Revista de Educación y Desarrollo, 8, 17-22. 
Webb, N. (1992). Assessment of student's knowledge of mathematics: Steps toward a theory. En D. Grouws (Ed.), Handbook of research on mathematics teaching and learning (pp. 81-93). Macmillan.

Wormeli, R. (2006). Fair isn't always equal: Assessing and grading in the differentiated classroom. National Middel School Association (NMSA).

RAÚl ALONSO RAMÍREZ ESCOBAR

Dirección: Departamento de Ciencias Básicas del TecNM / Instituto Tecnológico de Nogales, Ave. Tecnológico 911 C.P. 84065 Colonia Granja, H. Nogales, Sonora, México

Teléfono: (631) 159-0001, (631) 314-8436 +526311112479 